\title{
$P$-Determinant Regularization Method for Elliptic Boundary Problems
}

\section{Oscar A. Barraza}

Departamento de Matemática, U.N.L.P., c.c.172, 1990 La Plata, Argentina, and Universidad de San Andrés, c.c.1983, 1000 Buenos Aires, Argentina

Received: 3 February 1993/in revised form: 26 November 1993

Abstract. An expression for the $p$-determinant of the quotient of two differential elliptic operators with boundary conditions is given in terms of the boundary values of their solutions. Applications to physical examples are considered.

\section{Introduction}

An expression for the Fredholm determinant of the quotient of two elliptic operators defined on a closed manifold with boundary in terms of pseudodifferential operators defined on the boundary was given by Forman in [5]. In this paper, we aim to establish an analogous expression for the so called $p$-determinant of the quotient of the operators holding even in the case where it has not Fredholm determinant. This case is usually found in Quantum Physics where the $p$-determinant can be taken as a regularization technique for divergent determinants [9]. In order to describe it, let us recall some definitions.

A compact operator $A$ defined on a Hilbert space $H$ is an element of the $p^{\text {th }}$ Schatten class $\mathscr{T}_{p}$, for $p \geq 1$ an integer, if $|A|^{p}$ is a trace class operator, i.e. if

$$
\operatorname{Tr}\left(|A|^{p}\right)=\sum_{j=1}^{\infty} \mu_{j}^{p}(A)<\infty
$$

where $\mu_{j}(A)$, the singular values of $A$, are the eigenvalues of $|A|=\sqrt{A^{*} A}$. In particular $\mathscr{T}_{1}$ and $\mathscr{T}_{2}$ are the ideals of trace class and Hilbert-Schmidt operators on

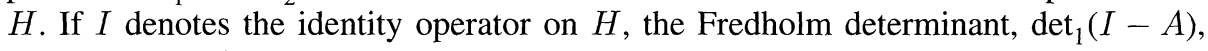
is defined as $\prod_{j=1}^{\infty}\left(1-\lambda_{j}\right)$, where $\left\{\lambda_{j}\right\}_{j}$ denotes the proper values of $A$ when $A$ is a trace class operator. The $p$-determinant of $I-A$ is defined, for $A \in \mathscr{F}_{p}$, as $[6,4,9]$ :

$$
\operatorname{det}_{p}(I-A)=\operatorname{det}_{1}\left\{I-(I-A) \exp \left[A+\frac{A^{2}}{2}+\ldots+\frac{A^{p-1}}{p-1}\right]\right\}
$$


or, equivalently [9]:

$$
\operatorname{det}_{p}(I-A)=\operatorname{det}_{1}\left(I-R_{p}(A)\right)
$$

where

$$
R_{p}(A)=(I-A) \exp \left[A+\frac{A^{2}}{2}+\ldots+\frac{A^{p-1}}{p-1}\right] .
$$

Note that $R_{p}(z)$ is an entire function on the complex plane $\mathbf{C}$ such that the expansion of its logarithm is obtained leaving out the first $(p-1)$-powers of the expansion of $\ln (1-z)$. It is easy to show that if $A \in \mathscr{T}_{p}$ then $R_{p}(A) \in \mathscr{T}_{1}$ [9]. We will be interested in differential operators of order $m>0$ defined from the $\mathscr{C}^{\infty}$ sections of a complex vector bundle $\left(E, M, \pi_{E}\right)$ to the ones of $\left(F, M, \pi_{F}\right)$, both bundles with fiber of dimension $k$, where $M$ is a $n$-dimensional compact manifold with boundary $X$. We will also be interested in pseudodifferential operators of order $s$ defined from the $\mathscr{C}^{\infty}$ sections of the complex vector bundle $\left(E_{\mid X}, X, \pi_{E}\right)$ to the ones of a vector bundle $\left(G, X, \pi_{G}\right)$ over $X$. We assume that the full symbols of these pseudo-differential operators have asymptotic expansions in homogeneous functions of the cotangent $\xi$-variables for $|\xi|>1$. This class of operators will be denoted by $I_{h}^{s}(X)$.

A $k \times k$ matrix $L$ of differential (respectively pseudodifferential) operators of order $m$ defined on $M$ (respectively on $X$ ) is (uniformly) elliptic in $M$ (respectively in $X$ ) if it has a principal symbol $\sigma_{0}(L)$ satisfying

$$
\left|\operatorname{det} \sigma_{0}(L)\right| \geq C|\xi|^{m k}, \quad \text { when } \quad|\xi|>N,
$$

for some positive constants $C$ and $N$.

In the case that $L$ is a matrix of differential operators of order $m$, a (non-necessarily orthogonal) projection onto the set of modified Cauchy of $\mathscr{C}^{\infty}$ functions belonging to the kernel of $L$ is given by the Calderón's projector $Q([2,7])$. This is a $\mathrm{km} \times \mathrm{km}$ matrix of pseudodifferential operators in the class $I_{h}^{0}(X)$ and its principal symbol $q$ depends only on $\sigma_{0}(L)$. It will be assumed that the $k m \times k m$ matrix $q$ has constant rank $r$. (This is always true for $n \geq 3$, see [2].)

A $r \times k m$ matrix $B$ of pseudodifferential operators belonging to $I_{h}^{0}(X)$,

$$
B: \underbrace{\mathscr{C}^{\infty}(X, E) \oplus \ldots \oplus \mathscr{C}^{\infty}(X, E)}_{m \text {-times }} \rightarrow \mathscr{C}^{\infty}(X, \tilde{G}),
$$

where $\tilde{G}$ is an $r$-dimensional vector bundle over $X$, with principal symbol $b$, is an elliptic boundary condition for the operator $L$ if the matrix $b q$ has constant rank equal to $r$ [2]. For such $L$ and $B$ the boundary problem $L_{B}=(L, B)$ is said to be elliptic. Actually, $L_{B}$ is the closed unbounded operator on $\mathscr{L}^{2}(M)$, obtained as the closure of $L$ acting on $\mathscr{C}^{\infty}$ sections of $E$ satisfying the boundary condition $B$ on $X[5,8]$. By $L_{B}^{-1}$ we mean the bounded operator which is the inverse of $L_{B}$, when it exists, and in this case we say that the problem $L_{B}$ is invertible.

We denote by $T$ the linear map which gives the Cauchy data values

$$
\begin{gathered}
T: \mathscr{C}^{\infty}(M, E) \rightarrow \underbrace{\mathscr{C}^{\infty}(X, E) \oplus \mathscr{C}^{\infty}(X, E) \oplus \ldots \oplus \mathscr{C}^{\infty}(X, E)}_{m \text {-times }} \\
u(x)=u\left(x^{\prime}, x_{n}\right) \mapsto T u(x)=\left(u\left(x^{\prime}\right), \partial_{\nu} u\left(x^{\prime}\right), \ldots, \partial_{\nu}^{m-1} u\left(x^{\prime}\right)\right),
\end{gathered}
$$

where $\nu$ is the unitary outward normal vector to the boundary $X$. For $x$ in a patch of $M$ with no empty intersection with $X$ we write $x=\left(x^{\prime}, x_{n}\right) \in M$ with $x^{\prime} \in X$ and $x_{n}$ the $X$-normal coordinate. 
The unique linear function

satisfying

$$
G(x, y): M \times M \rightarrow \operatorname{Hom}(F, E)
$$

(i) $L(G(x, y))=\delta(x, y), \delta(x, y)$ is the Dirac delta function; and

(ii) $T(G(x, y)) \in \operatorname{Ker}(B)$, (i.e. the Cauchy data values of $G(x, y)$ as function of $x$ belongs to the kernel of the boundary operator $B$ );

is called the Green's function for the boundary problem $L_{B}=(L, B)$ and it is the kernel function of the inverse $L_{B}^{-1}$.

When no confusion arises, $G(x, y)$ will be written as $L_{B}^{-1}(x, y)$.

Another linear map we shall consider is the Poisson's map

$$
\begin{aligned}
P_{B}: \mathscr{C}^{\infty}(X, \tilde{G}) & \rightarrow \mathscr{C}^{\infty}(M, E) \\
h & \mapsto P_{B}(h)=f,
\end{aligned}
$$

where $f$ satisfies $L f=0$ on $M$ and $B T f=h$ on $X$, when the data function $h$ belongs to $\operatorname{Im}(B)$.

For an elliptic problem $L_{B}=(L, B)$ the Poisson's map is an isomorphism between $\operatorname{Im}(B)$ and $\operatorname{Ker}(L)$, and verifies:

$$
B T P_{\left.B\right|_{\operatorname{Im}(B)}}=I_{\left.\right|_{\operatorname{Im}(B)}} \text { and } \quad P_{B} B T_{\left.\right|_{\operatorname{Ker}(L)}}=I_{\left.\right|_{\operatorname{Ker}(L)}} .
$$

For two boundary elliptic operators $A$ and $B$ a bijection $\Phi_{A B}$ from $\operatorname{Im}(B)$ onto $\operatorname{Im}(A)$ was defined in [5] as $\Phi_{A B}=A T P_{B}$.

According to (1) we obtain that $T P_{B}$ is a right inverse of $B$ and belongs to $I_{h}^{0}(X)$. The operators $T P_{B} B$ and $T P_{A} A$ also belong to $I_{h}^{0}(X)$ [7], just as $\Phi_{A B}$.

Our main result is the following:

Theorem 1. Let be $G$ an open subset of the complex plane. Let $\left\{L_{z}\right\}_{z \in G}$ be an analytic family in the $\mathscr{L}^{2}(M)$-norm of matrices of elliptic differential operators of order $m>0$, with the same principal symbol for all $z$, and defined from the $\mathscr{C}^{\infty}$ sections of a complex vector bundle $\left(E, M, \pi_{E}\right)$ to the ones of $\left(F, M, \pi_{F}\right)$, both with $k$-dimensional fibers. Let $z(t):[0,1] \rightarrow G$ be a differentiable path in $G$, and write $L_{t}=L_{z(t)}$.

If $A$ and $B$ are two boundary conditions such that $L_{t A}$ and $L_{t B}$ are elliptic invertible boundary problems for all $t \in[0,1]$, then for each $t \in[0,1]$ the pseudodifferential operator $L_{t B}^{-1} L_{t A} L_{0 A}^{-1} L_{0 B}$ has finite n-determinant and

$$
\operatorname{det}_{n}\left(L_{t B}^{-1} L_{t A} L_{0 A}^{-1} L_{0 B}\right)=\operatorname{det}_{n}\left(\Phi_{0 A B}^{-1} \Phi_{t A B}\right) .
$$

In particular, $I-L_{t B}^{-1} L_{t A} L_{0 A}^{-1} L_{0 B} \in \mathscr{T}_{n}$.

Remark. If we drop the hypothesis about the independence of the principal symbol on the parameter $t$ we cannot assert that $\left(I-L_{t B}^{-1} L_{t A} L_{0 A}^{-1} L_{0 B}\right)^{p}$ is a trace class operator for $p=n$; but the equality remains valid

$$
\operatorname{det}_{p}\left(L_{t B}^{-1} L_{t A} L_{0 A}^{-1} L_{0 B}\right)=\operatorname{det}_{p}\left(\Phi_{0 A B}^{-1} \Phi_{t A B}\right),
$$

for a value of $p$ such that the 1.h.s. is finite. have:

In particular, when $I-L_{t B}^{-1} L_{0 B}$ and $I-L_{0 A}^{-1} L_{t A}$ are trace class operators, we

$$
\begin{aligned}
\operatorname{det}_{1}\left(L_{0 B} L_{t B}^{-1}\right) \cdot \operatorname{det}_{1}\left(L_{t A} L_{0 A}^{-1}\right) & =\operatorname{det}_{1}\left(L_{0 B} L_{t B}^{-1} L_{t A} L_{0 A}^{-1}\right) \\
& =\operatorname{det}_{1}\left(L_{t B}^{-1} L_{t A} L_{0 A}^{-1} L_{0 B}\right) \\
& =\operatorname{det}_{1}\left(\Phi_{0 A B}^{-1} \Phi_{t A B}\right),
\end{aligned}
$$

as it was proved in [5] under additional hypothesis. 
Now, let us consider the case of a fixed operator $L$ and elliptic boundary conditions $A_{t}$ and $B_{t}$ depending on $t$. Let us assume that $A_{t}=A \mathscr{C}_{t}^{-1}$ and $B=B \mathscr{C}_{t}^{-1}$ with $A$ and $B$ two fixed elliptic conditions for $L$, and $\mathscr{C}_{t}$ is locally a $m \times m$ block matrix

$\mathscr{W}_{t}=\left(\begin{array}{ccccc}u_{t \mid X} & 0 & 0 & \ldots & 0 \\ \left(\begin{array}{l}1 \\ 0\end{array}\right) \partial_{\nu} u_{t \mid X} & \left(\begin{array}{l}1 \\ 1\end{array}\right) u_{t \mid X} & 0 & \ldots & 0 \\ \left(\begin{array}{l}2 \\ 0\end{array}\right) \partial_{\nu}^{2} u_{t \mid X} & \left(\begin{array}{l}2 \\ 1\end{array}\right) \partial_{\nu} u_{t \mid X} & \left(\begin{array}{c}2 \\ 2\end{array}\right) u_{t \mid X} & \ldots & 0 \\ \vdots & \vdots & \vdots & \ldots & \vdots \\ \left(\begin{array}{c}m-1 \\ 0\end{array}\right) \partial_{\nu}^{m-1} u_{t \mid X} & \left(\begin{array}{c}m-1 \\ 1\end{array}\right) \partial_{\nu}^{m-2} u_{t \mid X} & \left(\begin{array}{c}m-1 \\ 2\end{array}\right) \partial_{\nu}^{m-3} u_{t \mid X} & \cdots & \left(\begin{array}{c}m-1 \\ m-1\end{array}\right) u_{t \mid X}\end{array}\right)$

for $u_{t}$ a global section of the bundle $\operatorname{Iso}(E, E)$. (We have denoted by $u_{t \mid X}$ the restriction of the section $u_{t}$ to the boundary $X$.) Note that the inverse matrix of $\mathscr{W}_{t}$ is given by

$$
\mathscr{V}_{t}^{-1}=\left(\begin{array}{cccc}
{\left[u_{t}^{-1}\right]_{X}} & 0 & \cdots & 0 \\
\left(\begin{array}{c}
1 \\
0
\end{array}\right)\left[\partial_{\nu} u_{t}^{-1}\right]_{X} & \left(\begin{array}{l}
1 \\
1
\end{array}\right)\left[u_{t}^{-1}\right]_{X} & \cdots & 0 \\
\left(\begin{array}{l}
2 \\
0
\end{array}\right)\left[\partial_{\nu}^{2} u_{t}^{-1}\right]_{X} & \left(\begin{array}{l}
2 \\
1
\end{array}\right)\left[\partial_{\nu} u_{t}^{-1}\right]_{X} & \cdots & 0 \\
\vdots & \vdots & \cdots & \vdots \\
\left(\begin{array}{c}
m-1 \\
0
\end{array}\right)\left[\partial_{\nu}^{m-1} u_{t}^{-1}\right]_{X} & \left(\begin{array}{c}
m-1 \\
1
\end{array}\right)\left[\partial_{\nu}^{m-2} u_{t}^{-1}\right]_{X} & \cdots & \left(\begin{array}{c}
m-1 \\
m-1
\end{array}\right)\left[u_{t}^{-1}\right]_{X}
\end{array}\right)
$$

It is straightforward to see that $\mathscr{C}_{t}$ and $\mathscr{C}_{t}^{-1}$ belong to $I_{h}^{0}(X)$. We also have $\mathscr{C}_{t} T=T u_{t}$ and $\mathscr{C}_{t}^{-1} T=T u_{t}^{-1}$.

Without loss of generality we may suppose that $u_{0}=\mathrm{id}$, and then $\mathscr{C}_{0}=\mathrm{id}$. Thus the elliptic problem

$$
\begin{cases}L f=0 & \text { in } M \\ A \mathscr{U} b_{t}^{-1} T f=h & \text { in } X\end{cases}
$$

is equivalent to

$$
\begin{cases}L u_{t} g=0 & \text { in } M \\ A T g=h & \text { in } X\end{cases}
$$

where the dependence on the parameter $t$ has been transferred to the new operator $L_{t}=L u_{t}$. In this context, we write $L_{t A}=\left(L u_{t}\right)_{A}$ and $L_{t A}^{-1}=\left(L u_{t}\right)_{A}^{-1}=$ $u_{t}^{-1} \cdot L_{A \mathscr{R}_{t}^{-1}}^{-1}$. We have:

Theorem 2. Let $L$ be a $k \times k$-matrix of elliptic invertible differential operators of order $m>0$ which are defined from the $\mathscr{C}^{\infty}$ sections of a complex vector bundle $\left(E, M, \pi_{E}\right)$ to the ones of $\left(F, M, \pi_{F}\right)$ both with $k$-dimensional fibers.

Let be $\left\{A_{z}\right\}$ and $\left\{B_{z}\right\}$ two families of elliptic boundary conditions for $L$, considered as elliptic pseudodifferential operators in $I_{h}^{0}(X)$, analytically depending on the parameter $z$ with $z$ belonging to an open subset $G$ of the complex plane and let $z(t):[0,1] \rightarrow G$ a differentiable path in $G$.

Furthermore, let us suppose that there exists a family $\mathscr{C b}_{x}$ of smooth sections of the bundle $\operatorname{Iso}(E, E)$, analytically depeding on $z$, with $u_{0}=\mathrm{id}$ satisfying $A_{z}=A \mathscr{C}_{z}^{-1}$ and $B_{z}=B \mathscr{U}_{z}^{-1}$ for each $z \in G$, with $\mathscr{C}_{z}$ defined from $u_{z}$ as in (3a), and $A$ and 
$B$ two fixed elliptic boundary conditions for L. Let us put $u_{t}=u_{z(t)}, u_{t}^{-1}=u_{z(t)}^{-1}$, $\mathscr{W}_{t}=\mathscr{W}_{z(t)}$ and $\mathscr{W}_{t}^{-1}=\mathscr{W}_{z(t)}^{-1}$.

If

(i) the action by $u_{t}$ preserves principal symbol of each element of the Calderon's projection operator $Q$ for the operator $L$, or else

(ii) the principal symbols of $A$ and $B$ are the same, then

$$
\operatorname{det}_{n}\left\{u_{t}^{-1} L_{B \mathscr{R}_{t}^{-1}}^{-1} L_{A \mathscr{U}_{t}^{-1}} u_{t} L_{A}^{-1} L_{B}\right\}=\operatorname{det}_{n}\left(\Phi_{0 A B}^{-1} \Phi_{t A B}\right),
$$

where $\Phi_{t A B}=A \mathscr{U}_{t}^{-1} T P_{B \mathscr{U}_{t}^{-1}}$.

Remark. Note that hypothesis (i) is fulfilled if, for instance, this transformation commutes or anticommutes with the principal symbol of $L$ or $Q$.

The proofs of Theorems 1 and 2 are given in Sect. 2. Applications to physical examples are presented in Sect. 3. Technical lemmas about the regularity of the $p$-determinant are included in the appendix.

\section{Proof of Theorems 1 and 2}

We begin by proving the following lemma which gives an easy estimate for $p$.

Lemma 3. Under the hypothesis of Theorem 1, the operator $I-\Phi_{0 A B}^{-1} \Phi_{t A B}$ belongs to $I_{h}^{-1}(X)$ and $\left(I-\Phi_{0 A B}^{-1} \Phi_{t A B}\right)^{n}$ is trace class.

Proof. Let $a, b$, and $q$ be the principal symbols of the zero order pseudodifferential operators $A, B$ and $Q$ defined over $X$. (We drop the parameter $t$ because we are going to consider each member $L_{t}$ of the family separately.) The ellipticity condition for the problems $(L, A)$ and $(L, B)$ means that the principal symbols of $A Q$ and $B Q$, $a q$ and $b q$, are $r \times k m$ matrices with maximum rank $r$ [2]. We claim that the $r \times r$ matrices $a q q^{*} a^{*}, b q q^{*} b^{*}$, and $a q q^{*} b^{*}$ are invertible. Indeed, for the first two the proof is trivial. For the last one, note that $r=\operatorname{rank}(q)=\operatorname{rank}(b q)$, implies that $b$ is injective on the $\operatorname{Im}(q)$, and so $\operatorname{Ker}(q)=\operatorname{Ker}(b q)$. Analogously, $\operatorname{Ker}(q)=\operatorname{Ker}(a q)$. From this we have $\operatorname{Im}\left(q^{*} a^{*}\right)=\operatorname{Im}\left(q^{*} b^{*}\right)$ and, finally, $\operatorname{Im}\left(a q q^{*} b^{*}\right)=\operatorname{Im}\left(a q q^{*} a^{*}\right)$. This proves that $\operatorname{rank}\left(a q q^{*} b^{*}\right)=r$. Then $A Q Q^{*} B^{*}$ and $B Q Q^{*} B^{*}$ are zero order elliptic pseudodifferential operators on $X$. Each of them admits a right inverse because of the finite dimension of their kernels [2]. These right inverses will be denoted by $\left(A Q Q^{*} B^{*}\right)^{-1}$ and $\left(B Q Q^{*} B^{*}\right)^{-1}$. Their principal symbols are $\left(a q q^{*} b^{*}\right)^{-1}$ and $\left(b q q^{*} b^{*}\right)^{-1}$ respectively.

Now, let us define

$$
S_{A}=Q Q^{*} B^{*}\left(A Q Q^{*} B^{*}\right)^{-1} \text { and } S_{B}=Q Q^{*} B^{*}\left(B Q Q^{*} B^{*}\right)^{-1} .
$$

They satisfy:

$$
\begin{aligned}
& B S_{B}=I, \quad Q S_{B}=S_{B}, \\
& A S_{A}=I, \quad Q S_{A}=S_{A} .
\end{aligned}
$$

Since $S_{A}$ and $T P_{A}$ are right inverses of $A$, with $T$ the Cauchy data operator and $P_{A}$ the Poisson's one, and because the matrix $a$ is bijective on $\operatorname{Im}(q)$, it turns out that $T P_{A}=S_{A}+R_{A}$, where $R_{A}$ is an integral operator with infinitely differentiable kernel function [7]. Then, $\sigma_{0}\left(T P_{A}\right)=\sigma_{0}\left(S_{A}\right)$. Analogously we have $\sigma_{0}\left(T P_{B}\right)=\sigma_{0}\left(S_{B}\right)$ for the boundary condition $B$. 
Note that for every $t$ we have:

$$
\begin{aligned}
\sigma_{0}\left(\Phi_{t A B}^{-1}\right) & =\left(\sigma_{0}\left(\Phi_{t A B}\right)\right)^{-1}=\left(\sigma_{0}\left(A T P_{t B}\right)\right)^{-1} \\
& =\left(\sigma_{0}(A) \sigma_{0}\left(T P_{t B}\right)\right)^{-1}=\left(a . \sigma_{0}\left(S_{t B}\right)\right)^{-1} \\
& =\left(a q_{t} q_{t}^{*} b^{*}\left(b q_{t} q_{t}^{*} b^{*}\right)^{-1}\right)^{-1}=b q_{t} q_{t}^{*} b^{*}\left(a q_{t} q_{t}^{*} b^{*}\right)^{-1} \\
& =b . \sigma_{0}\left(S_{t A}\right)=\sigma_{0}(B) \sigma_{0}\left(T P_{t A}\right) \\
& =\sigma_{0}\left(B T P_{t A}\right)=\sigma_{0}\left(\Phi_{t B A}\right)
\end{aligned}
$$

So, the operator $\Phi_{0 A B}^{-1} \Phi_{t A B}$ is pseudodifferential, belongs to $I_{h}^{0}(X)$ and its principal symbol is

$$
\begin{aligned}
\sigma_{0}\left(\Phi_{t A B}\right) & =\sigma_{0}\left(\Phi_{0 B A}\right) \sigma_{0}\left(\Phi_{t A B}\right) \\
& =b q_{0} q_{0}^{*} b^{*}\left(a q_{0} q_{0}^{*} b^{*}\right)^{-1} a q_{t} q_{t}^{*} b^{*}\left(b q_{t} q_{t}^{*} b^{*}\right)^{-1}=\mathrm{id},
\end{aligned}
$$

since we are assuming that $\sigma_{0}\left(L_{t}\right)=\sigma_{0}\left(L_{0}\right)$, what implies that $q_{t}=\sigma_{0}\left(Q_{t}\right)=$ $\sigma_{0}\left(Q_{0}\right)=q_{0}[2,7]$. Consequently the principal symbol of $I-\Phi_{0 A B}^{-1} \Phi_{t A B}$ is the null matrix and so $I-\Phi_{0 A B}^{-1} \Phi_{t A B} \in I_{h}^{-1}(X)$. For $\operatorname{dim}(X)=n-1$, we conclude that

$$
I-\Phi_{0 A B}^{-1} \Phi_{t A B} \in \mathscr{T}_{n}
$$

and $\operatorname{det}_{n}\left(\Phi_{0 A B}^{-1} \Phi_{t A B}\right)$ is well defined. Q.E.D.

We recall two relations established in [5], that we will use below:

$$
\begin{aligned}
\frac{d}{d t} P_{t B} & =-L_{t B}^{-1} \frac{d}{d t}\left(L_{t}\right) P_{t B}, \\
P_{t A} A T L_{t B}^{-1} & =L_{t B}^{-1}-L_{t A}^{-1} .
\end{aligned}
$$

Lemma 4. For any positive integer $r$ we have:

$$
\left(L_{t A}^{-1}-L_{t B}^{-1}\right)\left(I-L_{t A} L_{0 A}^{-1} L_{0 B} L_{t B}^{-1}\right)^{r}=\left(I-P_{t B} B T P_{0 A} A T\right)^{r}\left(L_{t A}^{-1}-L_{t B}^{-1}\right) .
$$

Proof. It will be enough to prove the case $r=1$. For $r>1$ the proof will follow by induction on $r$.

Since, from (5.b),

$$
A T\left(L_{t A}^{-1}-L_{t B}^{-1}\right)=A T\left(-L_{t B}^{-1}\right) \quad \text { and } \quad B T\left(L_{t A}^{-1}-L_{t B}^{-1}\right)=B T\left(L_{t A}^{-1}\right) .
$$

Then

$$
\begin{aligned}
& \left(L_{t A}^{-1}-L_{t B}^{-1}\right)\left(I-L_{t A} L_{0 A}^{-1} L_{0 B} L_{t B}^{-1}\right) \\
& \quad=\left(L_{t A}^{-1}-L_{t B}^{-1}\right)-\left(L_{t A}^{-1}-L_{t B}^{-1}\right)\left(L_{t A} L_{0 A}^{-1} L_{0 B} L_{t B}^{-1}\right) \\
& \quad=\left(L_{t A}^{-1}-L_{t B}^{-1}\right)-P_{t B} B T L_{t A}^{-1} L_{t A} L_{0 A}^{-1} L_{0 B} L_{t B}^{-1} \\
& \quad=\left(L_{t A}^{-1}-L_{t B}^{-1}\right)-P_{t B} B T L_{0 A}^{-1} L_{0 B} L_{t B}^{-1} \\
& \quad=\left(L_{t A}^{-1}-L_{t B}^{-1}\right)-P_{t B} B T\left(L_{0 A}^{-1}-L_{0 B}^{-1}\right) L_{0 B} L_{t B}^{-1} \\
& \quad=\left(L_{t A}^{-1}-L_{t B}^{-1}\right)-P_{t B} B T P_{0 A} A T\left(-L_{0 B}^{-1}\right) L_{0 B} L_{t B}^{-1} \\
& =\left(L_{t A}^{-1}-L_{t B}^{-1}\right)-P_{t B} B T P_{0 A} A T\left(-L_{t B}^{-1}\right) \\
& \quad=\left(L_{t A}^{-1}-L_{t B}^{-1}\right)-P_{t B} B T P_{0 A} A T\left(L_{t A}^{-1}-L_{t B}^{-1}\right) \\
& \quad=\left(I-P_{t B} B T P_{0 A} A T\right)\left(L_{t A}^{-1}-L_{t B}^{-1}\right) . \quad \text { Q.E.D. }
\end{aligned}
$$

Now we are ready to give the proofs of Theorems 1 and 2 . 
Proof of Theorem 1. We know from Lemma 3 that each member of the analytic family $I-\Phi_{0 A B}^{-1} \Phi_{t A B}, t \in[0,1]$, is a pseudodifferential operator in the class $I_{h}^{-1}(X)$. (Recall that $X=\partial M$ is a $(n-1)$-dimensional compact manifold without boundary.) From (5.a), (5.b) and Lemma A.6 in the appendix, we have:

$$
\begin{aligned}
\partial_{t} \ln \operatorname{det}_{n}\left(\Phi_{0 A B}^{-1} \Phi_{t A B}\right) \\
\quad=-\operatorname{Tr}\left\{\left(I-\Phi_{0 A B}^{-1} \Phi_{t A B}\right)^{n-1}\left(\Phi_{0 A B}^{-1} \Phi_{t A B}\right)^{-1} \partial_{t}\left(I-\Phi_{0 A B}^{-1} \Phi_{t A B}\right)_{\mid \operatorname{Im}(B)}\right\} \\
\quad=\operatorname{Tr}\left\{\left(I-\Phi_{0 A B}^{-1} \Phi_{t A B}\right)^{n-1} \Phi_{t A B}^{-1} \Phi_{0 A B} \Phi_{0 A B}^{-1} \partial_{t}\left(\Phi_{t A B}\right)_{\mid \operatorname{Im}(B)}\right\} \\
\quad=\operatorname{Tr}\left\{\left(I-B T P_{0 A} A T P_{t B}\right)^{n-1} B T P_{t A} A T \partial_{t}\left(P_{t B}\right)_{\mid \operatorname{Im}(B)}\right\} \\
\quad=\operatorname{Tr}\left\{\left(I-B T P_{0 A} A T P_{t B}\right)^{n-1} B T P_{t A} A T\left(-L_{t B}^{-1} \cdot \partial_{t}\left(L_{t}\right) \cdot P_{t B}\right)_{\mid \operatorname{Im}(B)}\right\} \\
=\operatorname{Tr}\left\{\left(I-B T P_{0 A} A T P_{t B}\right)^{n-1} B T\left(P_{t A} A T\left(-L_{t B}^{-1}\right)\right) \cdot \partial_{t}\left(L_{t}\right) \cdot P_{t B \mid \operatorname{Im}(B)}\right\} \\
=\operatorname{Tr}\left\{\left(I-B T P_{0 A} A T P_{t B}\right)^{n-1} B T\left(L_{t A}^{-1}-L_{t B}^{-1}\right) \cdot \partial_{t}\left(L_{t}\right) \cdot P_{t B \mid \operatorname{Im}(B)}\right\} .
\end{aligned}
$$

Since $P_{t B}$ is an isomorphism betwee $\operatorname{Im}(B)$ and $\operatorname{Ker}\left(L_{t}\right)$, we have

$$
\begin{aligned}
& \partial_{t} \ln \operatorname{det}_{n}\left(\Phi_{0 A B}^{-1} \Phi_{t A B}\right) \\
& \quad=\operatorname{Tr}\left\{P_{t B}\left(I-B T P_{0 A} A T P_{t B}\right)^{n-1} B T\left(L_{t A}^{-1}-L_{t B}^{-1}\right) \cdot \partial_{t}\left(L_{t}\right)_{\mid \operatorname{Ker}\left(L_{t}\right)}\right\} \\
& \quad=\operatorname{Tr}\left\{\left(I-P_{t B} B T P_{0 A} A T\right)^{n-1} P_{t B} B T\left(L_{t A}^{-1}-L_{t B}^{-1}\right) \cdot \partial_{t}\left(L_{t}\right)_{\mid \operatorname{Ker}\left(L_{t}\right)}\right\} .
\end{aligned}
$$

By (5.b) and the definition of Green's function, $P_{t B} B T\left(L_{t B}^{-1}\right)=0$ and so,

$$
\begin{aligned}
\partial_{t} & \ln \operatorname{det}_{n}\left(\Phi_{0 A B}^{-1} \Phi_{t A B}\right) \\
& =\operatorname{Tr}\left\{\left(I-P_{t B} B T P_{0 A} A T\right)^{n-1}\left(L_{t A}^{-1}-L_{t B}^{-1}\right) \cdot \partial_{t}\left(L_{t}\right)_{\mid \operatorname{Ker}\left(L_{t}\right)}\right\} \\
& =\operatorname{Tr}\left\{\left(L_{t A}^{-1}-L_{t B}^{-1}\right)\left(I-L_{t A} L_{0 A}^{-1} L_{0 B} L_{t B}^{-1}\right)^{n-1} \cdot \partial_{t}\left(L_{t}\right)_{\mid \operatorname{Ker}\left(L_{t}\right)}\right\} .
\end{aligned}
$$

On the other hand

$$
\begin{aligned}
& \partial_{t} \ln \operatorname{det}_{n}\left(L_{t B}^{-1} L_{t A} L_{0 A}^{-1} L_{0 B}\right) \\
&=-\operatorname{Tr}\left\{\left(I-L_{t B}^{-1} L_{t A} L_{0 A}^{-1} L_{0 B}\right)^{n-1} L_{0 B}^{-1} L_{0 A} L_{t A}^{-1} L_{t B}\right. \\
&\left.\quad \times \partial_{t}\left(I-L_{t B}^{-1} L_{t A} L_{0 A}^{-1} L_{0 B}\right)_{\mid \operatorname{Ker}\left(L_{t}\right)}\right\} \\
&=\operatorname{Tr}\left\{\left(I-L_{t B}^{-1} L_{t A} L_{0 A}^{-1} L_{0 B}\right)^{n-1} L_{0 B}^{-1} L_{0 A} L_{t A}^{-1} L_{t B}\right. \\
&\left.\quad \times\left[-L_{t B}^{-1} \cdot \partial_{t}\left(L_{t}\right) L_{t B}^{-1} L_{t A} L_{0 A}^{-1} L_{0 B}+L_{t B}^{-1} \cdot \partial_{t}\left(L_{t}\right) L_{0 A}^{-1} L_{0 B}\right]_{\mid \operatorname{Ker}\left(L_{t}\right)}\right\} \\
&=\operatorname{Tr}\left\{\left(I-L_{t B}^{-1} L_{t A} L_{0 A}^{-1} L_{0 B}\right)^{n-1} L_{0 B}^{-1} L_{0 A} L_{t A}^{-1} L_{t B} L_{t B}^{-1} \cdot \partial_{t}\left(L_{t}\right)\right. \\
& \\
&\left.\times\left[L_{t A}^{-1}-L_{t B}^{-1}\right] L_{t A} L_{0 A}^{-1} L_{0 B \mid \operatorname{Ker}\left(L_{1}\right)}\right\} \\
&=\operatorname{Tr}\left\{\left[L_{t A}^{-1}-L_{t B}^{-1}\right] L_{t A} L_{0 A}^{-1} L_{0 B}\left(I-L_{t B}^{-1} L_{t A} L_{0 A}^{-1} L_{0 B}\right)^{n-1}\right. \\
&\left.\times L_{0 B}^{-1} L_{0 A} L_{t A}^{-1} \cdot \partial_{t}\left(L_{t}\right)_{\mid \operatorname{Ker}\left(L_{t}\right)}\right\} \\
&= \operatorname{Tr}\left\{\left[L_{t A}^{-1}-L_{t B}^{-1}\right]\left(I-L_{t A} L_{0 A}^{-1} L_{0 B} L_{t B}^{-1}\right)^{n-1} L_{t A} L_{0 A}^{-1}\right. \\
&\left.\times L_{0 B} L_{0 B}^{-1} L_{0 A} L_{t A}^{-1} \cdot \partial_{t}\left(L_{t}\right)_{\mid \operatorname{Ker}\left(L_{t}\right)}\right\} \\
&= \operatorname{Tr}\left\{\left[L_{t A}^{-1}-L_{t B}^{-1}\right]\left(I-L_{t A} L_{0 A}^{-1} L_{0 B} L_{t B}^{-1}\right)^{n-1} \partial_{t}\left(L_{t}\right)_{\mid \operatorname{Ker}\left(L_{t}\right)}\right\} .
\end{aligned}
$$

From (6) and (7), we see that

$$
\partial_{t} \ln \operatorname{det}_{n}\left(L_{t B}^{-1} L_{t A} L_{0 A}^{-1} L_{0 B}\right)=\partial_{t} \ln \operatorname{det}_{n}\left(\Phi_{0 A B}^{-1} F_{t A B}\right),
$$


for $t \in[0,1]$. In particular the 1.h.s. of (8) is finite.

By integrating from 0 to $t$ and taking exponentials, we get the theorem. Q.E.D.

The following lemma will be used for the proof of Theorem 2 .

Lemma 5. Let $q$ and $q_{t}$ be the principal symbols of the Calderón's projectors $Q$ and $Q_{t}$ for the operators $L$ and $L_{t}=L u_{t}$, respectively. Under the hypothesis of Theorem 2 we have $\left(q_{t}\right)_{h l}=u_{t}^{-1} q_{h l} u_{t}$, for all $h, l=1,2, \ldots, m$.

Proof. As in the introduction, let us consider the family of the operators $L_{t}=L u_{t}$, where $u_{t}$ is the nonsingular multiplicative operator. Its principal symbol is the matrix $u_{t}$.

Recall that the principal symbol of $L_{t}$ is given by $\sigma_{0}\left(L_{t}\right)=\sigma_{0}\left(L u_{t}\right)=$ $\sigma_{0}(L) \sigma_{0}\left(u_{t}\right)=\sigma_{0}(L) u_{t}$.

In each local chart $(\odot, \varphi)$, the principal symbol $q_{t}\left(x^{\prime}, x_{n}, \xi^{\prime}, \xi_{n}\right)$ can be computed by means of the expansion of the principal symbol of $L_{t}$ in powers of the conormal variable $\xi_{n}$ :

$$
\sigma_{0}\left(L_{t}\right)\left(x^{\prime}, x_{n}, \xi^{\prime}, \xi_{n}\right)=\sigma_{0}(L)\left(x^{\prime}, x_{n}, \xi^{\prime}, \xi_{n}\right) u_{t}=\sum_{j=0}^{m} \sigma_{m-j}(t)\left(x^{\prime}, x_{n}, \xi^{\prime}\right) \cdot \xi_{n}^{j},
$$

where each $\sigma_{m-j}(t)\left(x^{\prime}, x_{n}, \xi^{\prime}\right)=\sigma_{m-j}(0)\left(x^{\prime}, x_{n}, \xi^{\prime}\right) u_{t}$ and $\sigma_{0}(L)\left(x^{\prime}, x_{n}, \xi^{\prime}, \xi_{n}\right)=$ $\sum_{\jmath=0}^{m} \sigma_{m-j}(0)\left(x^{\prime}, x_{n}, \xi^{\prime}\right) \cdot \xi_{n}^{j}$. The symbol $q_{t}$ is an $m \times m$ block matrix, each of one is a $k \times k$ matrix given by (see $[2,7])$ :

$$
\begin{aligned}
\left(q_{t}\right)_{h l}= & \frac{i}{2 \pi} \int_{\Gamma}\left(\sigma_{0}\left(L_{t}\right)\left(x^{\prime}, x_{n}, \xi^{\prime}, \xi_{n}\right)\right)^{-1} \\
& \times \sum_{j=1}^{m} \sigma_{m-\jmath}(t)\left(x^{\prime}, x_{n}, \xi^{\prime}\right) \cdot \xi_{n}^{j-l+h-1} \cdot\left|\xi^{\prime}\right|^{l-h} d \xi_{n} \\
= & \frac{i}{2 \pi} \int_{\Gamma} u_{t}^{-1}\left(\sigma_{0}(L)\left(x^{\prime}, x_{n}, \xi^{\prime}, \xi_{n}\right)\right)^{-1} \\
& \times \sum_{j=1}^{m} \sigma_{m-j}(0)\left(x^{\prime}, x_{n}, \xi^{\prime}\right) u_{t} \cdot \xi_{n}^{j-l+h-1}\left|\xi^{\prime}\right|^{l-h} d \xi_{n} \\
= & u_{t}^{-1} q_{h l} u_{t},
\end{aligned}
$$

for $h l=1,2, \ldots, m$, where $\Gamma$ is any simple closed contour oriented clockwise and enclosing all poles of the integrand in $\operatorname{Im} \xi_{n}<0$ and $\sigma_{m-j}(t)$ are the symbols of the differential operators of order $m-j$ in the tangential variables $\xi^{\prime}$.

In particular, formula (9) tells us that the rank of the matrix $q_{t}$ does not depend on $t$, that is, it remains equal to $r$, the rank of $q$. Q.E.D.

Proof of Theorem 2. Recall that $\mathscr{C}_{t} T=T u_{t}$ and $\mathscr{C}_{t}^{-1} T=T u_{t}^{-1}$, for all $t$, and then $\mathscr{W}_{t} \mathscr{U}_{t}^{-1}=\mathscr{U}_{t}^{-1} \mathscr{W}_{t}=\mathrm{id}$.

Pick up the Poisson's map $P_{B_{t}}$ of the problem $L_{B_{t}}=\left(L, B_{t}\right)$. (See near Theorem 1.) Because of the nature of $u_{t}^{-1}$ and $\mathscr{C}_{t}^{-1}$ it is easy to show that $\operatorname{Im}(B)=\operatorname{Im}\left(B_{t}\right)$ and $\operatorname{Ker}\left(L_{t}\right)=u_{t}^{-1}(\operatorname{Ker} L)$. Let us consider $P_{t B}=u_{t}^{-1} P_{B_{t}}$. It 
results that $P_{t B}$ is the Poisson's map of the problem $L_{t B}=\left(L_{t}, B\right)$; recall that $L_{t}=L u_{t}$. In fact, $P_{t B}$ satisfies:

$$
\begin{aligned}
B T P_{t B \mid \operatorname{Im}(B)} & =B T u_{t}^{-1} P_{B_{t} \mid \operatorname{Im}\left(B_{t}\right)}=B \mathscr{U}_{t}^{-1} T P_{B_{t} \mid \operatorname{Im}\left(B_{t}\right)} \\
& =B_{t} T P_{B_{t} \mid \operatorname{Im}\left(B_{t}\right)}=I_{\mid \operatorname{Im}\left(B_{t}\right)}=I_{\mid \operatorname{Im}(B)},
\end{aligned}
$$

and

$$
\begin{aligned}
P_{t B} B T_{\mid \operatorname{Ker}\left(L_{t}\right)} & =u_{t}^{-1} P_{B_{t}} B T_{\mid u_{t}^{-1}(\operatorname{Ker}(L))}=u_{t}^{-1} P_{B_{t}} B T u_{t \mid \operatorname{Ker}(L)}^{-1} \\
& =u_{t}^{-1} P_{B_{t}} B \mathscr{U}_{t}^{-1} T_{\mid \operatorname{Ker}(L)}=u_{t}^{-1} P_{B_{t}} B_{t} T_{\mid \operatorname{Ker}(L)} \\
& =u_{t}^{-1} I_{\mid \operatorname{Ker}(L)}=I_{\mid \operatorname{Ker}\left(L_{t}\right)} .
\end{aligned}
$$

Now, the relationship between the Forman's maps $\Phi_{t A B}$ and $\Phi_{A_{t} B_{t}}$ associated to the problems $L_{t B}$ and $L_{t A}$, respectively, is given by:

$$
\Phi_{t A B}=A \mathscr{C}_{t}^{-1} T P_{B_{t}}=A_{t} T P_{B_{t}}=\Phi_{A_{t} B_{t}} .
$$

The proof continues now in the same way as the precedent one. The slight difference becomes when it is necessary to show that $I-\Phi_{0 A B}^{-1} \Phi_{t A B} \in I_{h}^{-1}(X)$. Because this operator belongs to $I_{h}^{0}(X)$, it is enough to see that its principal symbol is the null matrix. Indeed, when hypothesis (i) is satisfied, the proof of Lemma 3 applies. When hypothesis (ii) holds, $a=\sigma_{0}(A)=\sigma_{0}(B)=b$ and then

$$
\begin{aligned}
\sigma_{0}\left(I-\Phi_{0 A B}^{-1} \Phi_{t A B}\right) & =\mathrm{id}-\sigma_{0}\left(\Phi_{0 A B}^{-1}\right) \sigma_{0}\left(\Phi_{t A B}\right) \\
& =\mathrm{id}-b q q^{*} b^{*}\left(a q q^{*} b^{*}\right)^{-1} a q_{t} q_{t}^{*} b^{*}\left(b q_{t} q_{t}^{*} b^{*}\right)^{-1} \\
& =\mathrm{id}-\mathrm{id}=0 . \quad \text { Q.E.D. }
\end{aligned}
$$

\section{Some Applications}

\subsection{The Laplacian in the Disc}

Let us consider the differential operator

$$
L=-\Delta+\lambda^{2}
$$

acting on the functions $f(r, \theta)$ defined in the disc

$$
M=\{(r, \theta): 0 \leq r \leq R, 0 \leq \theta \leq 2 \pi\}
$$

with boundary conditions:

$$
\begin{aligned}
& A_{t} T f(R, \theta)=a \partial_{r} f(R, \theta)+(1-t a) f(R, \theta), \\
& B_{t} T f(R, \theta)=f(R, \theta) .
\end{aligned}
$$

If $u_{t}(r)$ is any smooth function such that $u_{t}^{-1}(R)=1$ and $\partial_{r} u_{t}^{-1}(R)=-t$, for $t>0$, the matrix $\mathscr{C}_{t}^{-1}$ given by $(3 \mathrm{~b})$ is $\mathscr{C}_{t}^{-1}=\left(\begin{array}{cc}1 & 0 \\ -t & 1\end{array}\right)$. We are interested in $t$ 's near $\frac{1}{a}$, because the first condition in (12) becomes a Neumann's condition type. 
We see that the boundary conditions $A_{t}$ and $B_{t}$ satisfy $A_{t}=A \mathscr{U}_{t}^{-1}, B_{t}=B \mathscr{U}_{t}^{-1}$, with $A$ and $B$ the $1 \times 2$ matrices $\left(\begin{array}{ll}1 & a\end{array}\right)$ and $\left(\begin{array}{ll}1 & 0\end{array}\right)$ respectively.

Then, we have:

$$
\begin{aligned}
& A T f(R, \theta)=a \partial_{r} f(R, \theta)+f(R, \theta), \\
& B T f(R, \theta)=f(R, \theta) .
\end{aligned}
$$

As in Theorem 2, (10) is transformed into:

$$
L_{t}=L u_{t}=-\Delta u_{t}+\lambda^{2}
$$

with boundary conditions $A$ and $B$.

If $\Phi_{t A B}$ is expanded in the basis $\left\{e^{i k \theta}\right\}_{k \in \mathbf{Z}}$ of the kernel of $L$, we have:

$$
\left\langle\Phi_{t A B} e^{\imath k^{\prime} \theta}, e^{\imath k \theta}\right\rangle=\left[(1-t a)+a \lambda \frac{I_{k}^{\prime}(\lambda R)}{I_{k}(\lambda R)}\right] \delta_{k k^{\prime}},
$$

where $I_{k}(z)$ is the modified $k$-Bessel function for $\lambda \neq 0$, and $I_{k}(z)=r^{|k|}$ for $\lambda=0$ and $k \in \mathbf{Z}$ [1]. The operator $I-\Phi_{0 A B}^{-1} \Phi_{t A B}$ is not trace class, but from Theorem 2 , we know that it is Hilbert-Schmidt. Note that hypothesis (i) of Theorem 2 is fulfilled because $\sigma_{0}(L)$ commutes with $u_{t}$.

Finally, for $\lambda \neq 0$ we obtain:

$$
\begin{aligned}
\operatorname{det}_{2}\left(u_{t}^{-1} L_{B^{\prime} L_{t}^{-1}}^{-1} u_{t} L_{A}^{-1} L_{B}\right)=\operatorname{det}_{2}\left(\Phi_{0 A B}^{-1} \Phi_{t A B}\right) \\
=\prod_{k=-\infty}^{\infty}\left\{1-\frac{t a}{1+a \lambda \frac{I_{k}^{\prime}(\lambda R)}{I_{k}(\lambda R)}}\right\} \exp \left\{\frac{t a}{1+a \lambda \frac{I_{k}^{\prime}(\lambda R)}{I_{k}(\lambda R)}}\right\},
\end{aligned}
$$

and, for $\lambda=0$,

$$
\begin{gathered}
\operatorname{det}_{2}\left(u_{t}^{-1} L_{B M_{t}^{-1}}^{-1} u_{t} L_{A}^{-1} L_{B}\right)=\operatorname{det}_{2}\left(\Phi_{0 A B}^{-1} \Phi_{t A B}\right) \\
=\prod_{k=-\infty}^{\infty}\left\{1-\frac{t a}{1+|k| \frac{a}{R}}\right\} \exp \left\{\frac{t a}{1+|k| \frac{a}{R}}\right\} .
\end{gathered}
$$

\subsection{Bosonic Field at Temperature $\frac{1}{\beta}>0$}

Let us consider the differential operator

$$
L=-\Delta-\partial_{t}^{2}+m^{2}
$$

on the three-dimensional manifold

$$
M=\left\{\left(r e^{\imath \theta}, t\right): 0 \leq r \leq R, 0 \leq \theta \leq 2 \pi, 0 \leq t \leq \beta\right\},
$$

with $t$ the temporal coordinate. $L$ acts on periodic functions in the $t$-direction satisfying $A \mathscr{C}_{s}^{-1} T f=0$ and $B \mathscr{C}_{s}^{-1} T f=0$ in $r=R$, with $A$ and $B$ the boundary conditions defined in (12) and the transformations $u_{s}$ and $\mathscr{Q} b_{s}$ as in the previous example. Now we have that $\Phi_{s A B}$ is diagonal in the basis of the functions $\left\{e^{i k \theta+i \omega_{n} s}\right\}_{n, k \in \mathbf{Z}}$ defined on the boundary of $M$ with $\omega_{n}=\frac{2 n \pi}{\beta}$. 
Since $\sigma_{0}(L)$ and $u_{s}$ commute, we have from Theorem 2 that the operator ( $I-$ $\left.\Phi_{0 A B}^{-1} \Phi_{s A B}\right)^{p}$ is trace class if $p=3=\operatorname{dim}(M)$, as it was shown by means of hard computation in [1]. Furthermore, we obtain:

$$
\begin{aligned}
& \operatorname{det}_{3}\left(u_{s}^{-1} L_{B \|_{s}^{-1}}^{-1} L_{A \psi_{s}^{-1}} u_{s} L_{A}^{-1} L_{B}\right)=\prod_{n=-\infty}^{\infty} \prod_{k=-\infty}^{\infty}\left\{1-\frac{s a}{1+a \lambda_{n} \frac{I_{k}^{\prime}\left(\lambda_{n} R\right)}{I_{k}\left(\lambda_{n} R\right)}}\right\} \\
& \quad \times \exp \left\{\frac{s a}{1+a \lambda_{n} \frac{I_{k}^{\prime}\left(\lambda_{n} R\right)}{I_{k}\left(\lambda_{n} R\right)}}+\frac{1}{2}\left(\frac{s a}{1+a \lambda_{n} \frac{I_{k}^{\prime}\left(\lambda_{n} R\right)}{I_{k}\left(\lambda_{n} R\right)}}\right)\right\} .
\end{aligned}
$$

\subsection{Variable External Field}

We now consider (18) and (19) with an external field $u_{s}(r, \theta)$ such that:

$$
\begin{aligned}
u_{s}(R, \theta) & =1, \\
u_{s} \partial_{r} u_{s}^{-1}(R, \theta) & =-s \sum_{l=-\infty}^{\infty} C_{l} e^{i l \theta}
\end{aligned}
$$

with $\mathscr{Z b}_{s}(R, \theta)$ obtained from $u_{s}$ as in (3a).

For instance, we can take $u_{s}(r, \theta)=e^{s g(r) f(\theta)}$, where $g(r)$ is a smooth function vanishing in $[0, \varepsilon)$, for some small $\varepsilon>0$ and behaving like $r-R$ in $(R-\varepsilon, R]$, and $f(\theta)$ is the $2 \pi$-periodic function given by $f(\theta)=\sum_{l=-\infty}^{\infty} C_{l} e^{\imath l \theta}$.

Since only boundary conditions were modified, we can consider the same basis as before for the kernel of $L$. As it was shown in [1] after a direct algebra it results:

$$
\left(\Phi_{0 A B}^{-1} \Phi_{s A B}\right)_{k^{\prime} k}^{n^{\prime} n}=\left\{\delta_{k^{\prime} k}-\frac{s a C_{k^{\prime}-k}}{1+a \lambda_{n} \frac{I_{k}^{\prime}\left(\lambda_{n} R\right)}{I_{k}\left(\lambda_{n} R\right)}}\right\} \delta^{n^{\prime} n} .
$$

For $\sigma_{0}(L)$ commutes $u_{s}$, hypothesis (i) is satisfied and so we get that $\operatorname{det}_{p}$ is finite if $p=\operatorname{dim}(M)=3$.

\subsection{Free Energy of a Four-Dimensional Chiral Bag}

As in [3], let us consider a theory of free massless fermions confined to a spherical cavity of fixed radius $R$ and interacting at the boundary with a hedgehog configuration of an external pionic field.

This theory can be described by the first order differential operator

$$
L=i \not \partial=i \sum_{\jmath=0}^{3} \gamma_{\jmath} \partial_{x_{\jmath}}
$$


acting on $t$-antiperiodic sections over the manifold $M=\left\{x \in \mathbf{R}^{4}:|x| \leq R\right\}$, for $t \in[0, \beta]$, with full symbol $\sigma(L)(x, \xi)=i \sum_{j=0}^{3} \gamma_{j} \xi_{j}$, where $x=\left(x_{0}, x_{1}, x_{2}, x_{3}\right)$, $\xi=\left(\xi_{0}, \xi_{1}, \xi_{2}, \xi_{3}\right), \mathrm{id}_{4}$ the $4 \times 4$ identity matrix and $\gamma_{j}$ are $4 \times 4$ Dirac matrices satisfying

$$
\begin{aligned}
& \left\{\gamma_{j}, \gamma_{k}\right\}=2 \delta_{j k} \mathrm{id}_{4}, \quad j, k=0,1,2,3 \\
& \gamma_{5}=i \gamma_{0} \gamma_{1} \gamma_{2} \gamma_{3}=\left(\begin{array}{cccc}
1 & 0 & 0 & 0 \\
0 & 1 & 0 & 0 \\
0 & 0 & -1 & 0 \\
0 & 0 & 0 & -1
\end{array}\right)
\end{aligned}
$$

The corresponding boundary conditions are:

$$
\begin{aligned}
& A T \psi=\frac{1}{2}\left(1+i \not h e^{-\imath \theta \tau \cdot \eta \gamma_{5}}\right) \psi=0 \quad \text { in } \quad r=R, \\
& B T \psi=\frac{1}{2}(1+i \not h) \psi=0 \quad \text { in } \quad r=R .
\end{aligned}
$$

with $\eta$ the outward normal to the bag surface and $\tau \cdot \eta=\sum_{j=1}^{3} \tau^{\jmath} \eta_{j}$, where $\tau^{\jmath}(j=$ $1,2,3)$ are the Pauli matrices. Let us take as $u_{s}$ the constant matrix $u_{s}=e^{-i s \tau \cdot \eta \gamma_{5}}$. It turns out that $\mathscr{W}_{s}$ is the same matrix.

We claim that the hypothesis (i) of Theorem 2 is fulfilled. To see this, note that in a local chart intersecting the boundary $\partial M=S^{3}$, with tangential coordinates $x^{\prime}=\left(x_{0}, x_{1}, x_{2}\right)$, cotangential $\xi^{\prime}=\left(\xi_{0}, \xi_{1}, \xi_{2}\right)$ and conormal $\xi_{3}$, we have from (23):

$$
\sigma(L)=\sigma_{0}(L)=a_{0}\left(x^{\prime}, \xi^{\prime}\right)+a_{1}\left(x^{\prime}, \xi^{\prime}\right) \xi_{3}
$$

with $a_{0}\left(x^{\prime}, \xi^{\prime}\right)=i \sum_{j=0}^{2} \gamma_{j} \xi_{j}$ and $a_{1}\left(x^{\prime}, \xi^{\prime}\right)=i \gamma_{3}$.

Following [2] we write:

$$
\begin{aligned}
q\left(x^{\prime}, \xi^{\prime}\right) & =\frac{i}{2 \pi} \int_{\Gamma}\left(\sigma_{0}(L)\right)^{-1} a_{1}\left(x^{\prime}, \xi^{\prime}\right) d \xi_{3} \\
& =\frac{i}{2 \pi} \int_{\Gamma}\left(a_{1}\left(x^{\prime}, \xi^{\prime}\right)^{-1} a_{0}\left(x^{\prime}, \xi^{\prime}\right)+\xi_{3} \mathrm{id}_{4}\right)^{-1} d \xi_{3}
\end{aligned}
$$

where $\Gamma$ is any simple close contour oriented clockwise and enclosing all poles of the integrand in $\operatorname{Im}\left(\xi_{3}\right)<0$.

Taking into account that $\gamma_{3}^{-1}=\gamma_{3}$, the integrand in (27) can be written as:

$$
\left(\sum_{j=0}^{2} \gamma_{3} \gamma_{j} \xi_{j}+\xi_{3} \mathrm{id}_{4}\right)^{-1}
$$

It is clear from (24) that it commutes with $\gamma_{5}$ and so $q$ commutes with $\mathscr{U}_{s}$.

Finally, we get that $\operatorname{det}_{4}\left(u_{s}^{-1} L_{B \mathscr{C _ { s }}-1}^{-1} L_{A \mathscr{U}_{s}^{-1}} u_{s} L_{A}^{-1} L_{B}\right)$ is finite and equal to $\operatorname{det}_{4}\left(\Phi_{0 A B}^{-1} \Phi_{s A B}\right)$. 


\section{Appendix: Some Technical Lemmas}

We prove in this appendix technical lemmas related to the differentiability of the trace and the $p$-determinant for bounded operators.

We will denote by $\mathscr{L}(H)$ the space of bounded linear operators on a separable Hilbert space $H$, by $\mathscr{T}_{p}$, the $p^{\text {th }}$ Schatten class operators on $H$ and by $\mathbf{C}$ the complex plane.

The demonstration techniques we shall use are inspired in [6].

4.I. The case of trace class operators $\left(\mathscr{T}_{1}\right)$

Lemma A.1. Let $A(z): G \rightarrow \mathscr{T}_{1}$ a holomorphic map from an open subset $G$ of $\mathbf{C}$ to the ideal $\mathscr{T}_{1}$ endowed with the norm of $\mathscr{B}(H)$. Suppose that the trace norm of $A(z),\|A(z)\|_{1}$ is bounded on every compact subset of $G$. Then the function $\operatorname{det}_{1}(I-A(z)): G \rightarrow \mathbf{C}$ is holomorphic.

Proof. Let $\left\{\Phi_{\jmath}\right\}_{1}^{\infty}$ be an orthogonal basis of $H$ and for each $n \geq 1$, let $P_{n}$ be the orthogonal projection onto the subspace spanned by $\left\{\Phi_{j}\right\}_{j=1}^{n}$.

Let us define $A_{n}(z)=P_{n} A(z) P_{n}$. Since, for each fixed $z \in G, A_{n}(z) \rightarrow A(z)$ for $n \rightarrow \infty$ in $\mathscr{T}_{1}$-norm,

$$
\operatorname{det}_{1}(I-A(z))=\lim _{n \rightarrow \infty} \operatorname{det}_{1}\left(I-A_{n}(z)\right),
$$

because det $_{1}$ is continuous in this norm.

For $A(z)$ is holomorphic on $G$,

$$
\operatorname{det}_{1}\left(I-A_{n}(z)\right)=\operatorname{det}\left(\delta_{j k}-\left(A(z) \Phi_{k}, \Phi_{j}\right)\right)_{j, k=1, \ldots, n}
$$

is holomorphic on $G$ and $\operatorname{det}_{1}(I-A(z))$ is a measurable function.

Then, for each $n$ we have:

$$
\operatorname{det}_{1}\left(I-A_{n}(z)\right)=\frac{1}{2 \pi i} \int_{|w-z|=r} \frac{\operatorname{det}_{1}\left(I-A_{n}(w)\right)}{w-z} d w,
$$

where the path $\{|w-z|=r\} \subset G$, is nonclockwise oriented. If we denote by $\lambda_{j}\left(A_{n}(z)\right)$ and $z_{j}\left(A_{n}(z)\right)$ the eigenvalues and the singular values of the operator $A_{n}(z)$ respectively, we have

$$
\begin{aligned}
\left|\operatorname{det}_{1}\left(I-A_{n}(z)\right)\right| & =\prod_{j=1}^{n}\left|1-\lambda_{j}\left(A_{n}(z)\right)\right| \\
& \leq \prod_{j=1}^{n} 1+\left|\lambda_{j}\left(A_{n}(z)\right)\right| \\
& \leq \prod_{j=1}^{n} 1+z_{j}\left(A_{n}(z)\right) \\
& \leq \prod_{j=1}^{n} e^{z_{\jmath}\left(A_{n}(z)\right)} \\
& =e^{\sum_{j=1}^{n} z_{j}\left(A_{n}(z)\right)} \\
& =e^{\left\|A_{n}(z)\right\|_{1}} \leq e^{\|A(z)\|_{1}},
\end{aligned}
$$

which is bounded by hypothesis for $z \in K$, being $K$ any compact subset of $G$. 
Finally, by applying the Lebesgue dominated convergence theorem we have from (A.1) the integral representation

$$
\operatorname{det}_{1}(I-A(z))=\frac{1}{2 \pi i} \int_{|w-z|=r} \frac{\operatorname{det}_{1}(I-A(w))}{w-z} d w
$$

which implies that $\operatorname{det}_{1}(I-A(z))$ is holomorphic in $G$. Q.E.D.

Lemma A.2. Under the hypothesis of Lemma A.1 we have:

(a) the derivative operator of $A(z)$ is trace class for all $z \in G$;

(b) the function $\operatorname{Tr}(A(z))$ is holomorphic in $G$;

(c) $\partial_{z}[\operatorname{Tr}(A(z))]=\operatorname{Tr}\left[\partial_{z} A(z)\right]$.

Remark. Since $\mathscr{T}_{1}$ endowed with the operator norm is not a closed subspace of $\mathscr{L}(H)$, the claim (a) is not obvious.

Proof. We will prove (a) by showing that the series $\sum_{j=1}^{\infty}\left\langle\partial_{z} A(z) \phi_{\jmath}, \phi_{\jmath}\right\rangle$ is absolutely convergent for all $z \in G$ and any orthonormal basis $\left\{\phi_{j}\right\}_{1}^{\infty}$ of $H$. By hypothesis, the functions $a_{j}(z)=\left\langle\partial_{z} A(z) \phi_{j}, \phi_{j}\right\rangle: G \rightarrow \mathbf{C}$ are holomorphic. Then the sequence $S_{n}(z)=\sum_{j=1}^{n} a_{j}(z)$ of holomorphic functions in $G$ tends to $\operatorname{Tr}(A(z))$ and is uniformly bounded in compact sets of $G$, because the hypothesis and the following inequality

$$
\left|S_{n}(z)\right| \leq \sum_{\jmath=1}^{n}\left|a_{\jmath}(z)\right| \leq \sum_{j=1}^{\infty}\left|a_{\jmath}(z)\right|=\|A(z)\|_{1} .
$$

For the path $\gamma=\{w-z \mid=r\} \subset G$, it is valid the integral representation

$$
S_{n}(z)=\frac{1}{2 \pi i} \int_{\gamma} \frac{S_{n}(w)}{w-z} d w
$$

and applying the Lebesgue dominated convergence theorem, we get:

$$
\operatorname{Tr}(A(z))=\frac{1}{2 \pi i} \int_{\gamma} \frac{\operatorname{Tr}(A(w))}{w-z} d w
$$

This shows that the function $\operatorname{Tr}(A(z))$ is holomorphic in $G$ and then

$$
\begin{aligned}
\partial_{z}[\operatorname{Tr}(A(z))] & =\lim _{n \rightarrow \infty} \partial_{z}\left(S_{n}(z)\right) \\
& =\lim _{n \rightarrow \infty} \sum_{j=1}^{n} \partial_{z}\left\langle A(z) \phi_{j}, \phi_{j}\right\rangle \\
& =\lim _{n \rightarrow \infty} \sum_{j=1}^{n}\left\langle\partial_{z} A(z) \phi_{j}, \phi_{j}\right\rangle \\
& =\sum_{j=1}^{+\infty}\left\langle\partial_{z} A(z) \phi_{j}, \phi_{j}\right\rangle,
\end{aligned}
$$


independently of the choice of the orthonormal basis $\left\{\phi_{1}\right\}_{1}^{\infty}$. In particular this formula is independent of any rearrangement of the basis and the series is absolutely convergent. So $\partial_{z} A(z)$ is trace class and the equality (A.2) can be written as

$$
\partial_{z}[\operatorname{Tr}(A(z))]=\operatorname{Tr}\left[\partial_{z} A(z)\right] \text {. Q.E.D. }
$$

Lemma A.3. Under the hypothesis of Lemma A.l we have:

$$
\partial_{z} \ln \left(\operatorname{det}_{1}(I-A(z))=-\operatorname{Tr}\left[(I-A(z))^{-1} \partial_{z}(A(z))\right] .\right.
$$

Proof. Arguing as in Lemma A.2 for the function $\ln \operatorname{det}_{1}(I-A(z))$, being $z$ such that $\operatorname{det}_{1}(I-A(z)) \neq 0$, we have:

$$
\ln \left[\operatorname{det}_{1}(I-A(z))\right]=\lim _{n \rightarrow \infty} \ln \left[\operatorname{det}_{1}\left(I-A_{n}(z)\right)\right]
$$

and

$$
\partial_{z} \ln \left[\operatorname{det}_{1}(I-A(z))\right]=\lim _{n \rightarrow \infty} \partial_{z} \ln \left[\operatorname{det}_{1}\left(I-A_{n}(z)\right)\right] .
$$

For the finite dimension matrices $A_{n}(z)$ of Lemma A.1 is valid that:

$$
\begin{aligned}
\partial_{z} \ln \left[\operatorname{det}_{1}\left(I-A_{n}(z)\right)\right] & =\partial_{z} \operatorname{Tr}\left[\ln \left(I-A_{n}(z)\right)\right] \\
& =\operatorname{Tr}\left[\left(I-A_{n}(z)\right)^{-1} \partial_{z}\left(I-A_{n}(z)\right)\right] \\
& =-\operatorname{Tr}\left[\left(I-A_{n}(z)\right)^{-1} \partial_{z} A_{n}(z)\right]
\end{aligned}
$$

and moreover $\left(I-A_{n}(z)\right)^{-1} \rightarrow(I-A(z))^{-1}$ in $\mathbb{Z}_{1}$.

Then by the continuity of the functional $\mathrm{Tr}$ in the ideal $\mathscr{T}_{1}$, we get

$$
\begin{aligned}
\partial_{z} \ln \left[\operatorname{det}_{1}(I-A(z))\right] & =\lim _{n \rightarrow \infty}-\operatorname{Tr}\left[\left(I-A_{n}(z)\right)^{-1} \partial_{z} A_{n}(z)\right] \\
& =-\operatorname{Tr}\left[(I-A(z))^{-1} \partial_{z} A(z)\right] . \quad \text { Q.E.D. }
\end{aligned}
$$

4.II. The case of operators in the Schatten's ideal $\pi_{p}, p>1$ )

Lemma A.4. Let $A(z): G \rightarrow \mathscr{T}_{p}$ a holomorphic map from an open subset $G$ of $\mathbf{C}$ to the ideal $\mathscr{T}_{p}$ endowed with the norm of $\mathscr{S}(H)$. Suppose that the $p^{\text {th }}$-Schatten ideal norm of $A(z),\|A(z)\|_{p}$ is bounded on every compact subset of $G$. Then the function $\operatorname{det}_{p}(I-A(z)): G \rightarrow \mathbf{C}$ is holomorphic.

Proof. Following [9], we have

$$
R_{p}(A(z))=I-(I-A(z)) e^{A(z)+\frac{(A(z))^{2}}{2}+\ldots+\frac{(A(z))^{p-1}}{p-1}}
$$

where $R_{p}(z)=1-(1-z) e^{z+\frac{z^{2}}{2}+\ldots+\frac{z^{p-1}}{p-1}}$ is an entire function. By hypothesis $A(z) \in \mathscr{Y}_{p}$, then $R_{p}(A(z)) \in \mathscr{T}_{1}$ and $\operatorname{det}_{p}(I-A(z))=\operatorname{det}_{1}\left(I-R_{p}(A(z))\right)$.

We are going to see that $R_{p}(A(z))$ satisfies the hypothesis of Lemma A.1. In order to show that $R_{p}(A(z)): G \rightarrow \mathscr{T}_{1}$ is a holomorphic function, we write

$$
R_{p}(A(z))=\frac{1}{2 \pi i} \int_{\Gamma_{z}} R_{p}(\lambda)(\lambda-A(z))^{-1} d \lambda,
$$

with $\Gamma_{z}$ such that the spectrum $\sigma(A(z))$ is contained in its interior. For instance, we can take $\Gamma_{z}=\{\lambda \in \mathbf{C} /|\lambda|=\mathbf{2}\|\mathbf{A}(\mathbf{z})\|\}$, nonclockwise oriented. 
If $r>0$ is such that $\{z \in \mathbf{C} /|\mathbf{z}|<\mathbf{r}\} \subset \mathbf{G}$ and $h \in \mathbf{C}$ with $|h| \leq r / 2$, then

$$
\begin{aligned}
& \frac{R_{p}(A(z+h))-R_{p}(A(z))}{h} \\
& \quad=\frac{1}{2 \pi i} \int_{\Gamma_{z}} R_{p}(\lambda) \frac{\left[(\lambda-A(z+h))^{-1}-(\lambda-A(z))^{-1}\right]}{h} d \lambda \\
& =\frac{-1}{2 \pi i} \int_{\Gamma_{z}} R_{p}(\lambda)(\lambda-A(z+h))^{-1} \frac{(A(z)-A(z+h))}{h}(\lambda-A(z))^{-1} d \lambda .
\end{aligned}
$$

By the mean value theorem between Banach spaces we have:

$$
\begin{aligned}
\|A(z)-A(z+h)\|_{H, H} & \leq|h| \max _{0 \leq t \leq 1}\left\|\partial_{z}(A(z+t h))\right\|_{H, H} \\
& \leq|h| \max _{|\nu| \leq 3 r / 2}\left\|\partial_{\nu}(A(\nu))\right\|_{H, H} \\
& =C|h| .
\end{aligned}
$$

Since $(\lambda-A(z+h))^{-1}=(\lambda-A(z))^{-1}\left[I+(A(z)-A(z+h))(\lambda-A(z))^{-1}\right]^{-1}$, we have

$$
\begin{aligned}
& \left\|(\lambda-A(z+h))^{-1}\right\|_{H, H} \\
& \quad \leq\left\|(\lambda-A(z))^{-1}\right\|_{H, H}\left\|\left[I+(A(z)-A(z+h))(\lambda-A(z))^{-1}\right]^{-1}\right\|_{H, H} \\
& \quad \leq\left\|(\lambda-A(z))^{-1}\right\|_{H, H}\left[1-\|\left(A(z)-A(z+h)\left\|_{H, H}\right\|(\lambda-A(z))^{-1} \|_{H, H}\right]^{-1}\right. \\
& \quad \leq 2\left\|(\lambda-A(z))^{-1}\right\|_{H, H}, \quad \text { for all } \lambda \in \Gamma_{z} .
\end{aligned}
$$

In fact, by the continuity of $A(z)$ in $\mathscr{L}(H)$, there exists $\delta>0$ such that if $|h|<\delta$ then $\|A(z)-A(z+h)\|_{H, H}>\frac{1}{2} \frac{1}{\max _{\lambda \in \Gamma_{z}}\left\|(\lambda-A(z))^{-1}\right\|_{H, H}}$. Taking $h$ such that $|h|<\min \{\delta, 3 r / 2\}$ we have $\|A(z)-A(z+h)\|_{H, H}\left\|(\lambda-A(z))^{-1}\right\|_{H, H}<\frac{1}{2}$ uniformly in $\lambda$ for $\lambda \in \Gamma_{z}$.

So, the function under the integral sign in (A.3) is bounded in $\mathscr{L}(H)$-norm by a $\lambda$-integrable function, for all $h$ close to zero. By Lebesgue dominated convergence theorem we have that the function $R_{p}(A(z))$ from $G$ to $H$ is holomorphic.

Moreover, writing $R_{p}(z)=z^{p} h(z)$, with $h(z)$ an entire function such that $h(0)=\frac{1}{p} \neq 0$, it results $R_{p}(A(z))=(A(z))^{p} h(A(z))$. Since $h(A(z))$ belongs to $\mathscr{B}(H)$ and $(A(z))^{p}$ is trace class, $R_{p}(A(z))$ is trace class and

$$
\left\|R_{p}(A(z))\right\|_{1} \leq\left\|(A(z))^{p}\right\|_{1}\|h(A(h))\|_{H, H} \leq\|A(z)\|_{p}^{p}\|h(A(z))\|_{H, H} .
$$

This inequality ensures us that $R_{p}(A(z))$ is uniformly bounded in every compact subset of $G$, because the first factor is so by hypothesis and the second one is a continuous function in $z$ restricted to a compact subset of $G$. Finally, by Lemma A.1 we conclude that the function $\operatorname{det}_{p}(I-A(z))$ is holomorphic. Q.E.D.

Lemma A.5. Under the hypothesis of Lemma A.4 we have:

(a) the derivative operator $\partial_{z} A(z)$ belongs to the ideal $\mathscr{T}_{p}$ for all $z \in G$;

(b) the function $\operatorname{Tr}\left[(A(z))^{p}\right]$ is holomorphic on $G$;

(c) $\partial_{z}\left[\operatorname{Tr}\left[(A(z))^{p}\right]\right]=p \operatorname{Tr}\left[(A(z))^{p-1} \partial_{z} A(z)\right]$. 
Proof. $A(z)$ holomorphic implies that in the $\mathscr{L}(H)$-norm,

$$
\partial_{z}(A(z))=\frac{1}{2 \pi i} \int_{\Gamma} \frac{A(w)}{(w-z)^{2}} d w
$$

where $\Gamma=\{|w-z|=r\} \subset G$ is a nonclockwise oriented path and $r>0$ is close to zero. From the hypothesis of boundness of $\|A(z)\|$ on compact subsets, we have:

$$
\begin{aligned}
\left\|\partial_{z}(A(z))\right\|_{p} & \leq \frac{1}{2 \pi} \sup _{\Gamma}\|A(z)\|_{p} \frac{2 \pi r}{r^{2}} \\
& =\frac{1}{r} \sup _{\Gamma}\|A(z)\|_{p}<\infty .
\end{aligned}
$$

Then $\partial_{z}(A(z)) \in \mathscr{T}_{p}$.

The claim (b) is a direct application of Lemma A.2. To prove (c) note that according to Lemma A.2 and the cyclic property of trace we have

$$
\begin{aligned}
\partial_{z} \operatorname{Tr}\left[A(z)^{p}\right] & =\operatorname{Tr}\left[\partial_{z}(A(z))^{p}\right] \\
& =\operatorname{Tr}\left[\sum_{j=1}^{p} A(z)^{j-1} \partial_{z}(A(z)) A(z)^{p-\jmath}\right] \\
& =\sum_{j=1}^{p} \operatorname{Tr}\left[A(z)^{j-1} \partial_{z}(A(z)) A(z)^{p-\jmath}\right] \\
& =\sum_{\jmath=1}^{p} \operatorname{Tr}\left[A(z)^{p-1} \partial_{z}(A(z))\right] \\
& =p \operatorname{Tr}\left[A(z)^{p-1} \partial_{z}(A(z))\right] . \quad \text { Q.E.D. }
\end{aligned}
$$

Lemma A.6. Under the hypothesis of Lemma A.4 we have

$$
\partial_{z} \ln \operatorname{det}_{p}(I-A(z))=-\operatorname{Tr}\left[(I-A(z))^{-1}(A(z))^{p-1} \partial_{z}(A(z))\right] .
$$

Proof. For all $z \in G$ such that $I-A(z)$ is invertible, we have

$$
\ln \operatorname{det}_{p}(1-A(z))=\ln \operatorname{det}_{1}\left(1-R_{p}(A(z))\right),
$$

with $R_{p}(A(z))$ as before.

From Lemmas A.3 and A.4, we get

$$
\begin{aligned}
\partial_{z} \ln \operatorname{det}_{p}(I-A(z)) & =\partial_{z} \ln \operatorname{det}_{1}\left(I-R_{p}(A(z))\right) \\
& =-\operatorname{Tr}\left[\left(I-R_{p}(A(z))\right)^{-1} \partial_{z}\left(R_{p}(A(z))\right)\right] .
\end{aligned}
$$

Let $\left\{\phi_{j}\right\}_{j=1}^{\infty}$ be an orthonormal basis of $H$, and $P_{n}$ be the orthogonal projection onto the subspace generated by $\left\{\phi_{j}, j=1, \ldots, n\right\}$. Then $A(z)=\lim _{n \rightarrow \infty} A_{n}(z)$ in the norm of $\mathscr{T}_{p}$ being $A_{n}(z)=P_{n} A(z) P_{n}$.

Note that for all positive integer $r$ such that $1 \leq r \leq p, A_{n}(z)^{r} \rightarrow A(z)^{r}$ for $n \rightarrow \infty$ in the ideal $\mathscr{T}_{p / r}$-norm because $A(z)^{r} \in \overline{\mathscr{F}}_{p / r}$ and $A_{n}(z)^{r}=P_{n} A(z)^{r} P_{n}$. 
On the other hand, if $h(z)$ is an $z$-entire function, for $\Gamma$ a path which surrounds the spectrum of $A(z)$, and $z \in G$, we have:

$$
\begin{aligned}
& \left\|h\left(A_{n}(z)\right)-h(A(z))\right\|_{H, H} \\
& \quad=\left\|\frac{1}{2 \pi i} \int_{\Gamma} h(\lambda)\left[\left(\lambda-A_{n}(z)\right)^{-1}-(\lambda-A(z))^{-1}\right] d \lambda\right\|_{H, H} \\
& \quad \leq \frac{1}{2 \pi} \int_{\Gamma}|h(\lambda)|\left\|\left[\left(\lambda-A_{n}(z)\right)^{-1}-(\lambda-A(z))^{-1}\right]\right\|_{H, H}|d \lambda| \\
& \quad \leq \frac{1}{2 \pi} \int_{\Gamma}|h(\lambda)|\left\|\left(\lambda-A_{n}(z)\right)^{-1}\right\|_{H, H}\left\|A(z)-A_{n}(z)\right\|_{H, H}\left\|(\lambda-A(z))^{-1}\right\|_{H, H}|d \lambda| \\
& \quad \leq\left(\frac{1}{\pi} \int_{\Gamma}|h(\lambda)|\left\|(\lambda-A(z))^{-1}\right\|_{H, H}^{2}|d \lambda|\right)\left\|A(z)-A_{n}(z)\right\|_{H, H} \underset{n \rightarrow \infty}{\longrightarrow} 0,
\end{aligned}
$$

since $\left\|\left(\lambda-A_{n}(z)\right)^{-1}\right\|_{H, H} \leq 2\left\|(\lambda-A(z))^{-1}\right\|_{H, H}$ for large $n$.

So, $h\left(A_{n}(z)\right)$ tends to $h(A(z))$ in the $H$-norm for $n \rightarrow \infty$. Applying the triangular inequality we obtain

$$
R_{p}(A(z))=\lim _{n \rightarrow \infty} R_{p}\left(a_{n}(z)\right) \text { in } \mathscr{T}_{1}
$$

because $R_{p}(A(z))=g(A(z))$, being $g(z)=z^{p} h(z)$, with $h(z)$ an entire function.

Then

$$
\partial_{z} \ln \operatorname{det}_{p}(I-A(z))=-\lim _{n \rightarrow \infty} \operatorname{Tr}\left\{\left[I-R_{p}\left(A_{n}(z)\right)\right]^{-1} \partial_{z}\left[R_{p}\left(A_{n}(z)\right)\right]\right\} .
$$

Now, for each positive integer $n$ we have

$$
\begin{aligned}
\operatorname{Tr}\{ & {\left.\left[I-R_{p}\left(A_{n}(z)\right)\right]^{-1} \partial_{z}\left[R_{p}\left(A_{n}(z)\right)\right]\right\}=\operatorname{Tr}\left\{\left[I-g\left(A_{n}(z)\right)\right]^{-1} \partial_{z}\left[g\left(A_{n}(z)\right)\right]\right\} } \\
= & \operatorname{Tr}\left\{\left(I-A_{n}(z)\right)^{-1} e^{-A_{n}(z)-\frac{1}{2} A_{n}(z)^{2}-\ldots-\frac{1}{p-1} A_{n}(z)^{p-1}}\right. \\
& \left.. \partial_{z}\left[I-\left(I-A_{n}(z)\right) e^{A_{n}(z)+\frac{1}{2} A_{n}(z)^{2}+\ldots+\frac{1}{p-1} A_{n}(z)^{p-1}}\right]\right\} \\
= & -\operatorname{Tr}\left\{\left(I-A_{n}(z)\right)^{-1} e^{-A_{n}(z)-\ldots-\frac{1}{p-1} A_{n}(z)^{p-1}}\right. \\
\times & {\left[-\partial_{z}\left(A_{n}(z)\right) \cdot e^{A_{n}(z)+\ldots+\frac{1}{p-1} A_{n}(z)^{p-1}}\right.} \\
& +\left(I-A_{n}(z)\right) \sum_{\jmath=1}^{p-1} e^{A_{n}(z)+\ldots+\frac{1}{\jmath-1} A_{n}(z)^{\jmath-1}} \\
& \left.\left.. \partial_{z}\left(e^{\frac{1}{\jmath} A_{n}(z)^{\jmath}}\right) e^{\frac{1}{\jmath+1} A_{n}(z)^{\jmath+1}+\ldots+\frac{1}{p-1} A_{n}(z)^{p-1}}\right]\right\} \\
= & \operatorname{Tr}\left[\left(I-A_{n}(z)\right)^{-1} \partial_{z}\left(A_{n}(z)\right)\right]-\sum_{\jmath=1}^{p-1} \operatorname{Tr}\left[e^{-\frac{1}{\jmath} A_{n}(z)^{\jmath}} \partial_{z}\left(e^{\frac{1}{\jmath} A_{n}(z)^{\jmath}}\right)\right] .
\end{aligned}
$$


(We have used the cyclic property of traces for finite dimensional matrices to get the last equality.)

Applying the Cauchy formula to the finite dimensional matrices $e^{\frac{A_{n}(z)^{3}}{J}}$, it is straightforward to see that:

$$
\begin{aligned}
\operatorname{Tr}\left[e^{\frac{-A_{n}(z)^{\jmath}}{\jmath}} \partial_{z}\left(e^{\frac{A_{n}(z)^{\jmath}}{\jmath}}\right)\right] & =\operatorname{Tr}\left[e^{\frac{-A_{n}(z)^{\jmath}}{\jmath}} e^{\frac{A_{n}(z)^{\jmath}}{\jmath}} A_{n}(z)^{\jmath-1} \partial_{z}\left(A_{n}(z)\right)\right] \\
& =\operatorname{Tr}\left[A_{n}(z)^{\jmath-1} \partial_{z}\left(A_{n}(z)\right)\right]
\end{aligned}
$$

Then we have:

$$
\begin{aligned}
\operatorname{Tr} & \left\{\left[I-R_{p}\left(A_{n}(z)\right)\right]^{-1} \partial_{z}\left[R_{p}\left(A_{n}(z)\right)\right]\right\} \\
& =\operatorname{Tr}\left[\left(I-A_{n}(z)\right)^{-1} \partial_{z}\left(A_{n}(z)\right)\right]-\sum_{j=1}^{p-1} \operatorname{Tr}\left[A_{n}(z)^{j-1} \partial_{z}\left(A_{n}(z)\right)\right] \\
& =\operatorname{Tr}\left[\left(\left(I-A_{n}(z)\right)^{-1}-\sum_{j=1}^{p-1} A_{n}(z)^{\jmath-1}\right) \partial_{z}\left(A_{n}(z)\right)\right] \\
& =\operatorname{Tr}\left[\left(I-A_{n}(z)\right)^{-1} A_{n}(z)^{p-1} \partial_{z}\left(A_{n}(z)\right)\right] .
\end{aligned}
$$

(In the last equality, Taylor's formula was utilized with rest.)

It is easy to verify that

$$
\left(I-A_{n}(z)\right)^{-1} \underset{n \rightarrow \infty}{\longrightarrow}(I-A(z))^{-1} \text { in the norm of } \mathscr{L}(H),
$$

and that

$$
A_{n}(z)^{p-1} \underset{n \rightarrow \infty}{\longrightarrow} A(z)^{p-1} \text { in the norm of the ideal } \mathscr{T}_{p / p-1} .
$$

On the other hand, since

$$
\partial_{z}\left(A_{n}(z)\right)=\partial_{z}\left(P_{n} A(z) P_{n}\right)=P_{n} \partial_{z}(A(z)) P_{n},
$$

we have

$$
\partial_{z}\left(A_{n}(z)\right) \underset{n \rightarrow \infty}{\longrightarrow} \partial_{z}(A(z)) \text { in the norm of the ideal } \mathscr{T}_{p} .
$$

Putting it all together, we get

$$
\left(1-A_{n}(z)\right)^{-1} A_{n}(z)^{p-1} \partial_{z}\left(A_{n}(z)\right) \underset{n \rightarrow \infty}{\longrightarrow}(1-A(z))^{-1} A(z)^{p-1} \partial_{z}(A(z))
$$

in the trace norm.

From this, (A.4) and (A.6), we finally obtain:

$$
\begin{aligned}
\partial_{z} \operatorname{lndet}_{p}(1-A(z)) & =-\lim _{n \rightarrow \infty} \operatorname{Tr}\left[\left(1-A_{n}(z)\right)^{-1} A_{n}(z)^{p-1} \partial_{z}\left(A_{n}(z)\right)\right] \\
& =-\operatorname{Tr}\left[\left(1-A_{n}(z)\right)^{-1} A_{n}(z)^{p-1} \partial_{z}\left(A_{n}(z)\right)\right],
\end{aligned}
$$

because of the continuity of the trace in the trace norm. Q.E.D.

Acknowledgements. The author is grateful to H. Falomir, R. E. Gamboa Saraví, M. Santangelo, of Departamento de Física, Universidad Nacional de La Plata, and very specially to M. A. Muschietti and J. E. Solomin, of Departamento de Matemática, Universidad Nacional de La Plata, for their ideas and suggestions. 


\section{References}

1. Barraza, O., Falomir, H., Gamboa Saraví, R.E., Santangelo, E.M.: P-determinants and boundary values. J. Math. Phys. 33, 6 (1992)

2. Calderón, A.P.: Pseudodifferential operators and elliptic boundary value problems I. Publicaciones del I.A.M., núm. 1, Buenos Aires 1976

3. De Francia, M., Falomir, H., Santangelo, E.M.: Free energy of a four-dimensional chiral bag. Phys. Rev. D 45, 6 (1992)

4. Dunford, N., Schwartz, J.: Linear operators. II. New York: Interscience 1958

5. Forman, R.: Functional determinants and geometry. Invent. Math. 88, 447-493 (1987)

6. Gohberg, I.C., Krein, M.G.: Introduction to the theory of linear nonselfadjoint operators, vol. 18 (Translation of Mathematical Monographs). Providence, R.I.: American Mathematical Society 1969

7. Hörmander, L.: The analysis of the linear partial differential operators. III. Berlin, Heidelberg, New York: Springer 1985

8. Seeley, R.: The resolvent of an elliptic boundary problem. Am. J. Math. 91, 889-920 (1969)

9. Simon, B.: Trace ideals and their applications. London Mathematical Society, Lecture Notes Series 35. Cambridge: Cambridge University Press 1979

Communicated by H. Araki 